\title{
Rise of Designer Babies: In Defiance with Nature! CRISPR-Cas9-A New Gene Editing Tool
}

${ }^{1}$ Simranpreet Kaur, ${ }^{2}$ Madhu Nagpal

\begin{abstract}
A new genome-editing tool, clustered regularly interspaced short palindromic repeat (CRISPR)-Cas9, could transform the field of biology, and a recent news on the birth of a first gene-edited baby has created a lot of media hype. But, why such a big hype for CRISPR-Cas9, as scientists have already been tinkering with genomes for decades. The answer is in the technology of CRISPR, which is far better than older techniques for gene splicing and editing, doing the work with unprecedented precision, efficiency, and flexibility and just raising the fear of the development of designer babies. Germline editing means editing sperm, eggs, or embryos, i.e., altering the DNA of future generations even before birth, putting a question mark on the line of morality and ethics.
\end{abstract}

Keywords: CRISPR babies, CRISPR-Cas9, Ethical considerations, Gene editing, Genetic manipulation.

How to cite this article: Kaur S, Nagpal M. Rise of Designer Babies: In Defiance with Nature! CRISPR-Cas9-ANew Gene Editing Tool. Curr Trends Diagn Treat 2018;2(2):130-132.

Source of support: Nil

Conflict of interest: None

\section{INTRODUCTION}

In the post-genomic era, which is fast expanding farther, the future definition of biological function of genome sequences and their transcripts in development and disease is a huge challenge. For understanding the mechanism underlying development, normal physiology, and disease, targeted mutagenesis based on homologous recombination has been a powerful tool. Genome sequencing has been a finding of the beginning of the millennium. The research has not halted at that point, but further research on the micromanipulation of genes has taken place. A recent breakthrough in genome engineering technology based on the class of RNA-guided endonucleases is clustered regularly interspaced short palindromic repeat (CRISPR)-associated Cas9 \{CRISPR-Cas9\}, which is revolutionizing biology and medical studies.

${ }^{1}$ Embryologist cum Counsellor, ${ }^{2} \mathrm{Head}$

1,2 Department of Obstetrics and Gynaecology, Sri Guru Ram Das Institute of Medical Sciences \& Research, Amritsar, Punjab, India

Corresponding Author: Simranpreet Kaur, Department of Obstetrics and Gynaecology, Sri Guru Ram Das Institute of Medical Sciences \& Research, Amritsar, Punjab, India, Phone: 0183-2870200, e-mail: simranpreet_sandhu@yahoo.com
CRISPR-Cas9 is a new therapeutic intervention for genetic disorders. Cas9 is an enzyme that snips DNA, and CRISPR is a collection of DNA sequences that tell Cas9 where to snip. It is a unique technology that enables geneticists and medical researchers to edit parts of genome by cutting out, replacing, or adding parts to the DNA sequence.

\section{CRISPR-CAS9 TECHNOLOGY}

CRISPR refers to the unique organization of short, partially palindromic repeated DNA sequences found in the genomes of bacteria and other microorganisms. While seemingly innocuous, CRISPR sequences are a crucial component of the immune systems of these simple life forms. Like eukaryotes, bacterial cells are invaded by viruses. If a viral infection threatens a bacterial cell, the CRISPR immune system can thwart the attack by destroying the genome of the invading virus. The genome of the virus includes genetic material that is necessary for the virus to continue replicating. Thus, by destroying the viral genome, the CRISPR immune system protects bacteria from ongoing viral infection. It is currently the most simplest versatile and precise method of genetic manipulation and therefore causes a buzz in science world. ${ }^{1}$

\section{HOW DOES IT WORK}

The CRISPR-Cas9 system consists of two key molecules that introduce a mutation (change) into the DNA. An enzyme called Cas9 acts as a pair of molecular scissors, which can cut the two strands of DNA at a specific location in the genome, so that bits of DNA can be added or removed. A piece of RNA called guide RNA (gRNA) consists of a small piece of predesigned RNA sequences located within a longer RNA scaffold. The scaffold part binds to DNA and the predesigned sequence guides Cas 9 to the right part of the genome. This makes sure that the Cas9 enzyme gets cut at the right point in the genome. The gRNA is designed to find and bind to a specific sequence in the DNA. The gRNA has RNA bases that are complementary to those of the target DNA sequences in the genome. Cas9 follows the gRNA to the same location in the DNA sequence and makes a cut across both strands of the DNA. At this stage, the cell recognizes that the DNA is damaged and tries to repair it. 
Several "gene editing" technologies have been developed to improve gene targeting methods, including CRISPR-Cas systems, transcription activator-like effector nucleases (TALENs), and zinc-finger nucleases (ZFNs). The CRISPR-Cas9 system currently stands out as the fastest, cheapest, and most reliable system for "editing" genes. $^{2}$

Till now, experimenting with DNA needed sophisticated labs, years of experience, and millions of dollars. But, the use of CRISPR has changed all that. To set up a CRISPR editing capability, a lab needs to only order an RNA fragment and purchase shelf chemicals and enzymes costing only a few dollars. As CRISPR is cheap and easy to use, it has both revolutionized and democratized genetic research. Thousands of labs worldwide are experimenting with CRISPR-based editing projects. CRISPR-Cas9 has a lot of potential as a tool for treating a range of medical conditions that have a genetic component, hemoglobinopathies (thalassemias and sickle cell disease), hepatitis B, AIDS, various muscular dystrophies, and complex genetic syndromes. ${ }^{3}$

Many of the applications involve editing the genomes of somatic cells, but there has been a lot of interest in and debate about the potential to edit germline cells. Carrying out gene editing in germline cells is currently illegal in the UK, the US, and most other countries. But, China has taken the lead largely as it lacks the regulations and moral constraints other countries follow.

Chinese scientists in 2014 announced the successful production of monkeys that had been genetically modified at the embryonic stage. ${ }^{4}$ Later, in 2015, another group of researchers in China published a research article detailing the first ever attempt to edit human embryos. ${ }^{5}$ The attempt failed, but it shocked the world. Then, in April 2016, another group of researchers in China reported the successful modification of the genome of a human embryo to make it resistant to HIV. ${ }^{6}$

Recently, a scientist from the Southern University of Science and Technology in Shenzhen, China, has claimed that he has succeeded in creating world's first genetically edited babies. The Associated Press was told that twin girls were born earlier in the month of November this year. The embryos were edited using CRISPR technology to remove the CCR5 (C-C chemokine receptor type 5) gene. Disabling this gene would make the babies more resistant to HIV, cholera, and smallpox.

The CCR 5 gene codes for a protein that allows HIVthe AIDS causing virus-to enter a cell. According to researchers, embryos were edited for seven couples affected by HIV, and one successful pregnancy led to the birth of twin girls using this treatment. The CRISPRCas9 gene-editing tool was used to modify the embryos, before they were implanted into their respective mother's uterus. Eleven embryos were used in six attempts before the twin pregnancy was achieved. The technique could potentially prevent HIV from being passed on.

Couples could choose whether to use edited or unedited embryos for pregnancy attempts. While the news has caused an outcry in the scientific community, there is no independent confirmation of the claim, and it has not been published in a journal yet. ${ }^{7}$

\section{DOES IT SURPASS A SERIOUS BOUNDARY?}

Humans still know too little about predicting the broader effects of altering or disabling a gene. About a year ago, bioethicists did not see the widespread use of CRISPRCas9 in the next two decades even for the prevention of genetic diseases, let alone for designer babies. This kind of genetic editing is banned in most countries including the US and the UK as the technology is still in its experimental stages. The DNA changes can pass to future generations potentially with unforeseen side effects. Genetic modification techniques could lead to the elimination of devastating genetic disorders like Parkinson's disease and Down syndrome before a person is even born and could help families in which both the parents carry genes that increase the risk of a birth defect. The removal of these genes could significantly lower the risk of genetically influenced birth defects and could eventually lead to the removal of these genes from the entire family tree over time. However, critics warn that interfering with the blueprints of life to prenatally destroy diseases could lead to unintended genetic consequences that are even worse than whatever disease we are trying to cure. ${ }^{8}$

In the era of the 1960s, researchers imagined naively that, with time, they would understand, with increasing precision, the role of each gene in making humans. The foundation of genetics for decades, biology's central dogma, was the hypothesis that each gene codes for a single protein. Knowing the correspondences, tools will be devised which will be useful not only for research, but also for curing and preventing diseases with genetic basis and may be for augmenting human evolution.

The one-gene-one-protein central dogma, though it continues to pervade our common beliefs about genetics, underwent conversion when scientists realized that many proteins comprise several polypeptides, each of which was coded for by a gene. The dogma therefore became one gene, one polypeptide. But, what sounded the entire dogma's death knell was the discovery in the early 1970s that a single gene can code for more than one protein. The discovery that the human genome contains only about 30,000 genes to code for some 90,000 proteins brought that home. What made our understanding spectacularly 
inadequate is the discovery in 2000 that a single gene can potentially code for tens of thousands of proteins. ${ }^{9}$

We do not know the limits of the new technologies, cannot guess what lifetime effects a single gene alteration will have on a single individual, and have no idea at all about what effects the alteration of genes in sperm or ova or a fetus will have on future generations. For these reasons, we have no knowledge of whether a particular modification of the human germline will be ultimately catastrophic and no basis for considering that tampering with heritable genes can be humane or ethical. Scientists have recently learned that the approach to gene editing can inadvertently wipe out and rearrange large swaths of DNA in ways that may impair human health. This follows recent studies showing that CRISPR-edited cells can inadvertently trigger cancer. ${ }^{10}$

CRISPR-Cas9 had been tried in adults as a possible way to cure cancer and treat deadly diseases. But, the difference is that these changes are limited to only one person. By prenatally making genetic changes, we take the risk of changing the genetics of a family tree forever. And it is possible that as this technology improves, it will be used for more than just disease prevention. ${ }^{11}$ Researchers suggest that even if we focus initially on improving health, it could lead to nontherapeutic genetic enhancement, such as strength, beauty, gender, and intelligence. India has already been gender imbalanced; for every 107 males, there are 100 females. Given the disposition of parents to favor males, preference for fairer skin, higher intelligence, and even extra height and strength, there will soon be competition to create perfect children with these technologies. What if the embryo editing techniques spiral out of control allowing parents to custom order a designer baby? How this will shape the future of human race and at what cost only time will tell?

Humans are on the verge of finally being able to modify their own evolution, and they have a tendency to make the impossible possible. We know that human evolution has not stopped. In fact, as it turns out, we may end up having more control of it now than ever before. We have entered in an era where genetic manipulation is no longer a science-fiction novelty; it is a reality of the present. $^{12}$

We do not yet know what the future will look like, but one thing is certain, gene editing is here to stay, and it is only going to get more sophisticated. The question that whether this newfound superpower will be used in a responsible way that will benefit the planet and its people still remains to be answered in future.

\section{REFERENCES}

1. Wilkinson R, Wiedenheft B. A CRISPR method for genome engineering. F1000Prime REPORTS 2014 Jan;6:3, doi: 10.12703/P6-3.

2. Doudna J, editor. Jennifer Doudna: we can now edit our DNA, but lets do it wisely. TedTalk 2015. Videofileretrievedfrom https://www.ted.com/talks/jennifer_doudna_we_can_ now_edit_our_dna_but_let_do_it_wisely

3. Regaldo A. CRISPR gene editing to be tested on people by 2017, says Editas. MIT Technology Review 2015.

4. Shen H. First monkeys with customized mutations born. Nature/NEWS 2014 Jan 30, doi: 10.1038/nature.2014.14611.

5. Cyranoski D, Reardon S. Chinese Scientists genetically modify human embryos. Nature/NEWS 2015 April 22.

6. Regaldo A. Rewriting life. Chinese researchers experiment with making HIV-proof embryos. MIT Technology Review 2016 April 8.

7. Yong Ed. A reckless and needless use of gene editing on Human embryos. The Atlantic: Science 2018 Nov 26.

8. Darnovsky M. Aslippery slope tohuman germline modification. Nature 2013;499:127.

9. Wadhwa V. We are challenging our evolution. Hindustan Times: Editorial 2018 Nov 28:14.

10. Cyranoski D. Ethics of embryo editing divides scientists. Nature 2015;519:272.

11. Fogleman S, Santana C, Bishop C, et al. CRISPR/Cas9 and mitochondrial gene replacement therapy: promising techniques and ethical considerations. Am J Stem Cells 2016 Aug;5(2):39-52.

12. Yang X. Applications of CRISPR-Cas 9 mediated genome engineering. Yang Military Medical Research 2015;2:11, doi: 10.1186/s40779-015-0038-1. 